\title{
Contribution of Myocardial Perfusion's Tomoscintigraphy in the Management of Coronarian Disease in Senegal: About 42 Cases
}

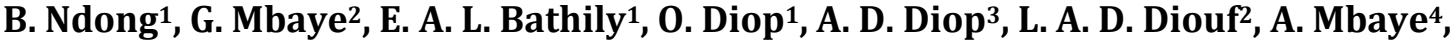 \\ R. S. Senghor1, M. Soumboundou², A. R. Djiboune'2, P. M. Sy², N. Badji ${ }^{3}$, A. Dia', M. S. Djigo', \\ M. Mbodj' ${ }^{1}$ O. Ndoye1, M. Diarra² ${ }^{2}$ A. Kane ${ }^{4}$, S. Seck-Gassama1 \\ ${ }^{1}$ Department of Nuclear Medicine, General Hospital of Grand Yoff (GHOGY), Dakar, Senegal \\ ${ }^{2}$ Laboratory of Physical and Pharmaceutical Biophysics, Faculty of Medicine Pharmacy and Odontology, \\ Cheikh Anta Diop University, Dakar, Senegal \\ ${ }^{3}$ Radiology Department, Fann University Hospital, Dakar, Senegal \\ ${ }^{4}$ Cardiology HOGGY General Hospital of Grand Yoff (GHOGY), Dakar, Senegal \\ ${ }^{5}$ Training and Health Research of Thiès, Thiès, Sénégal \\ Email: ndongboucar73@yahoo.fr, mbaygo@yahoo.fr
}

How to cite this paper: Ndong, B., Mbaye, G., Bathily, E.A.L., Diop, O., Diop, A.D., Diouf, L.A.D., Mbaye, A., Senghor, R.S., Soumboundou, M., Djiboune, A.R., Sy, P.M., Badji, N., Dia, A., Djigo, M.S., Mbodj, M., Ndoye, O., Diarra, M., Kane, A. and SeckGassama, S. (2017) Contribution of Myocardial Perfusion's Tomoscintigraphy in the Management of Coronarian Disease in Senegal: About 42 Cases. Open Journal of Biophysics, 7, 175-182.

https://doi.org/10.4236/ojbiphy.2017.74013

Received: June 26, 2017

Accepted: July 29, 2017

Published: August 1, 2017

Copyright $\odot 2017$ by authors and Scientific Research Publishing Inc. This work is licensed under the Creative Commons Attribution International License (CC BY 4.0). http://creativecommons.org/licenses/by/4.0/

\begin{abstract}
The aim of the study was to evaluate the contribution to moscintigraphy of myocardial perfusion (TSMP) with ${ }^{99 \mathrm{~m}} \mathrm{Tc}$-sestamibi in the management of suspected or known coronary patients's population. It included 42 patients with an average age of $57.02 \pm 9.77$ years with extremes ranging from 36 to 77 years. The pre-test probability in 38 patients was high in 7 patients (18.42\%), intermediate in 29 patients $(76.31 \%)$ and low in 2 patients (5.26\%). Patients with a high pre-test probability had a positive scintigraphy. Among the 26 patients who underwent transthoracic ultrasound (TTU), 10 with normal kinetics on trans-thoracic ultrasound (TTU) had positive scintigraphy. For 16 patients with TTU abnormalities, 6 had negative scintigraphy and 10 had positive scintigraphy. The TSMP was positive for 28 patients (66.66\%). Among these patients, 16 had pure ischemia, $57.14 \%$ (16/28), 7 pure necrosis $(25 \%)$, and 5 both necrosis and ischemia. Myocardial perfusion scintigraphy remains a very contributory consideration in the management of coronary disease. This is synchronized with the electrocardiogram (ECG) and is an important diagnostic and prognostic tool for coronary artery disease. It also provides a good indication of coronary angiography.
\end{abstract}

\section{Keywords}

Tomoscintigraphy, Myocardial Perfusion, Coronary Artery Disease 


\section{Introduction}

Coronary disease is the leading cause of death in developed countries and the second in developing countries [1] [2]. According to the World Health Organization (WHO), ischemic heart disease is a major concern worldwide with $12.2 \%$ of all deaths. It predicts that these heart diseases will be the leading cause of death in Africa by 2025 [3]. Tomoscintigraphy of myocardial perfusion (TSMP) allows the evaluation of myocardial perfusion related to rest and/or after a challenge test of myocardial ischemia (stress test or pharmacological stress). It is a reference method for the detection of ischemia and myocardial necrosis. It is also used in therapeutic evaluation (after coronary angioplasty or coronary bypass grafting), in the assessment of prognosis for a myocardial viability study after thallium myocardial infarction [4]. However, its low prescription in our regions contrasts with the relatively high frequency of its indications. The objective of this work was to show, through a local study, the role of MPTS in the detection and management of myocardial perfusion disorders, with a view to raising awareness.

\section{Material and Method}

We performed a retrospective, mono-centric, cross-sectional study over 2 years (2014-2016), including 42 patients, 16 men and 26 women, with a mean age of $57.02 \pm 9.77$ years, referred for myocardial perfusion exploration. The sex ratio was 1.62 for women. The following table (Table 1 ) shows the distribution of patients by sex and age range.

The chosen protocol was variable (one day or two days). For the one-day (stress-rest) protocol, the dose of the radioactive tracer $\left({ }^{99 \mathrm{~m}} \mathrm{Tc}-\mathrm{MIBI}\right.$ or ${ }^{99 \mathrm{~m}} \mathrm{Tc}$ - tetrofosmin) was 8 - $10 \mathrm{mci}$ for effort acquisition and $16-20 \mathrm{mci}$ for resting , 4 hours later. For the two-day protocol, a dose of $15 \mathrm{mci}$ was injected during each examination. The stress test was performed on a treadmill according to the Bruce protocol. Pharmacological stress was not used in our study [5]. Tomographic acquisitions were carried out 15 to 30 minutes after injection of the radiotracer using a dual head TEMP (2007) gamma camera equipped with the Interview XP software with a LEHR (Low Energy High Resolution) collimator.

Table 1. Distribution of patients by sex and age range.

\begin{tabular}{cccc}
\hline Age range & Men (\%) & Women (\%) & Total (\%) \\
\hline$[30-40[$ & 7.14 & 0 & 7.14 \\
{$[40-50[$} & 2.38 & 7.14 & 9.52 \\
{$[50-60[$} & 16.67 & 28.57 & 45.24 \\
{$[60-70[$} & 4.77 & 23.81 & 28.58 \\
{$[70-80[$} & 7.14 & 2.38 & 9.52 \\
Total (\%) & 38.10 & 61.90 & 100 \\
\hline
\end{tabular}


The technique is low in irradiation, the marker used $\left({ }^{99 \mathrm{~m}} \mathrm{Tc}, \mathrm{T}=6 \mathrm{~h}\right)$ has a physical period long enough to allow the recording of images and short enough to minimize irradiation of the patient and the entourage.

\section{Results}

\subsection{Scintigraphy and Perfusion Abnormalities}

\section{1) Artifacts}

Images of attenuation were found in 7 patients and most often interested the anterior territory. More than half of the patients with mitigation were women.

\section{2) The quality of the images and lack of perfusion}

The images obtained were of good quality in more than half of the cases ( 31 out of 42 , or $73.3 \%$ ); of mean quality in 7 patients $(16 \%)$ and of poor quality in 10.7\% (4 patients) (Figures 1-3).

\subsection{Myocardial Perfusion Scintigraphy and Pre-Test Probability}

The pre-test probability was calculated according to the table of Diamond and Forrester [6] and was based on age, sex and especially the symptomatology. In our study, this pre-test probability was high in 7 patients $(18.42 \%)$, intermediate in 29 patients $(76.31 \%)$ and in 2 patients (5.26\%). All four patients who did not undergo this test were known coronary patients. All patients with a high pre-test probability had a positive scintigraphy. There was a statistically significant correlation $(p=0.01)$ between positive perfusion myocardial scintigraphy and pre-test probability (Table 2 ).
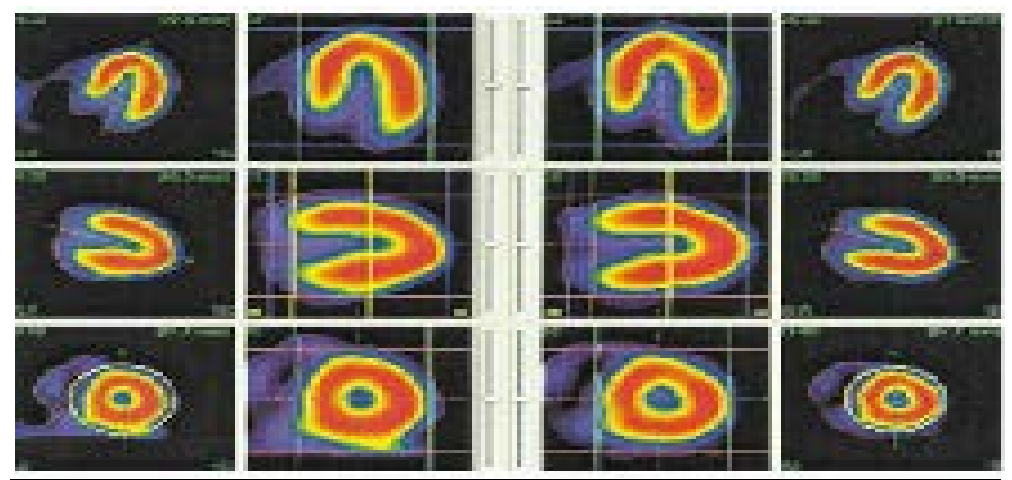

Figure 1. Normal myocardial scintigraphy of a 45 -year-old patient with atypical chest pain. 1st line: large horizontal axis section, 2nd line: large vertical axis section, 3rd line: minor axis section.

Table 2. Distribution of myocardial scintigraphy results according to the pre-test probability.

\begin{tabular}{ccc}
\hline Pre-test probability & $\begin{array}{c}\text { negative myocardial } \\
\text { perfusion scintigraphy }\end{array}$ & $\begin{array}{c}\text { Positive myocardial } \\
\text { perfusion scintigraphy }\end{array}$ \\
\hline High & $0 \%$ & $18.42 \%$ \\
Intermediate & $27.62 \%$ & $48.70 \%$ \\
Low & $5.26 \%$ & $0 \%$ \\
\hline
\end{tabular}




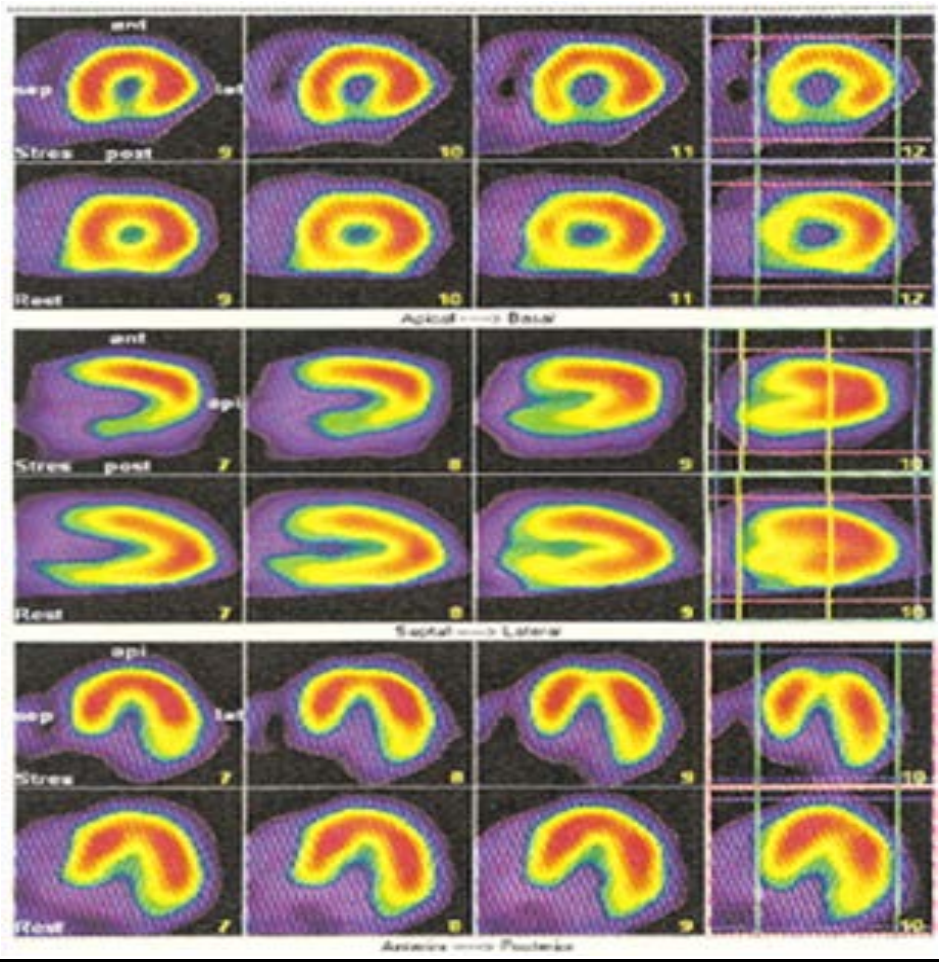

Figure 2. Scintigraphy of a 62-year-old patient with reversible hypofixation of the postero-inferior segment (ischemia). At the left: Two first lines: small axis cuts with hypofixation of the lower wall to the reversible effort at rest. 3rd and 4th lines: large vertical axis cuts showing hypofixation of the reversible postero-inferior wall at rest. 5th and 6th lines: large horizontal axis cuts with no anomalies.

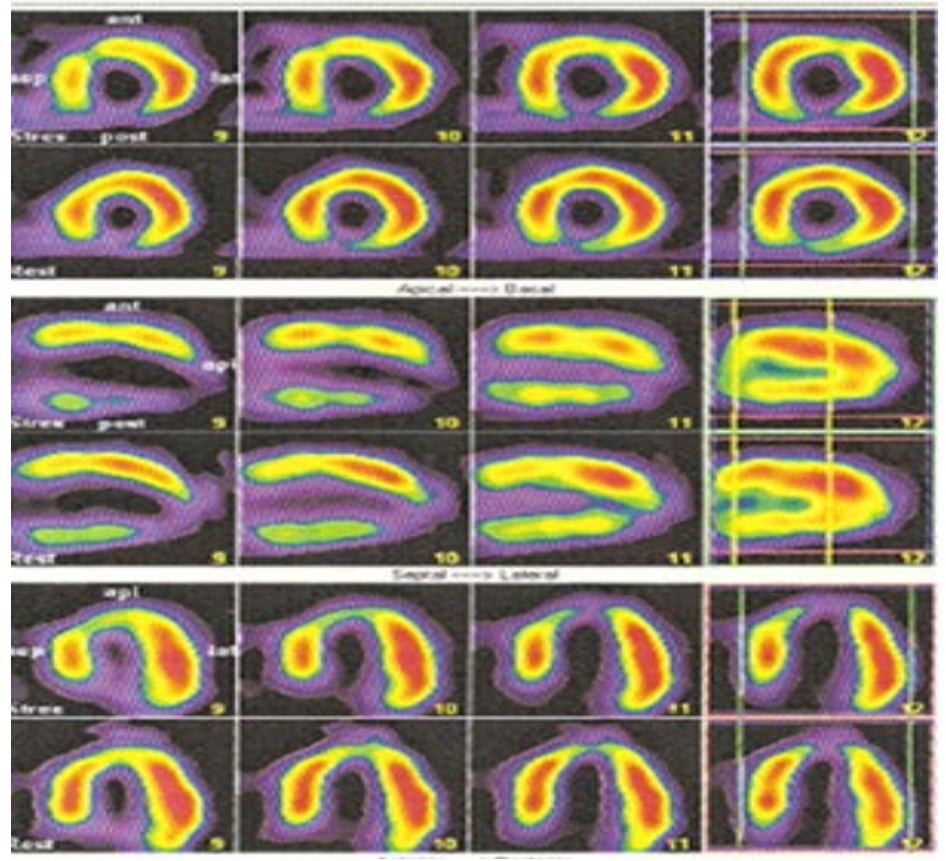

Figure 3. Scintigraphy of a 64-year-old hypertensive patient with reversible hypofixation of the anterior wall (Ischemia) and irreversible hypofixation of the apical two-thirds of the lower wall overflowing on the apex (necrosis). 


\subsection{Myocardial Scintigraphy and Trans-Thoracic Echocardiography (TTE)}

Among the 42 patients, 26 received a TTE of $61.90 \%$. Ten of these 26 patients $(10 / 26,38.46 \%)$ had a normal TTE and $16(16 / 26,61.53 \%)$ had an abnormal TTE. Six patients $(6 / 16,37.5 \%)$ had negative scintigraphy and 10 patients $(10 / 16$, $62.5 \%)$ had positive scintigraphy for the 16 patients $(61.53 \%)$ with TTE abnormalities.

\subsection{Abnormalities of the Scintigraphy}

The TSMP was positive in 28 patients (66.66\%). Among these patients, 16 had pure ischemia, $57.14 \%(16 / 28), 7$ patients had pure necrosis $(25 \%)$ and 5 patients had both necrosis and ischemia. In patients with pure ischemia, only two had a number of affected segments $>3 / 17$. In cases of pure necrosis, with the exception of one case, the number of segments reached was $\geq 3 / 17$. The number of affected segments was $>3 / 17$ in all patients with both necrosis and ischemia. The lesions were mostly found at the lower, anterior and apex walls. Separate damage to the septal and lateral walls was not observed in our study.

\section{Discussion}

\subsection{Stimulation Test}

We chose the exercise on treadmill as stimulation test. This stress test is physiological, without major constraint and is favorable to the evaluation of the perfusion of the myocardium.

The prognostic variables associated with the exercise electrocardiogram can be obtained. It was the same for the onset of chest pain in relation to the intensity of exercise, the maximum heart rate reached and the corresponding blood pressure. Thus, Ladenheim et al. [4] showed that the maximum heart rate reached with exercise is a significant independent predictor of cardiac events. This information cannot be obtained with pharmacological stress. A failure to increase blood pressure above $130 \mathrm{~mm} \mathrm{Hg}$ or even a drop in blood pressure of less than $10 \mathrm{mmHg}$ indicates left ventricular dysfunction or extensive ischemia [7]. In the literature, an exercise test conducted beyond 10 Mets shows an excellent prognosis regardless of the presence of coronary artery disease. On the contrary, the inability to reach 6 Mets is in favor of increased cardiovascular mortality.

\subsection{Artifacts}

In our study, technetium was the only marker used in all patients and the quality of the images obtained was good in nearly 3/4 of the patients. These images are most often due to a superposition of the diaphragm or the breasts [8]. These artifacts can be avoided by breast bandaging or if necessary in procubitus. These results are different from those of Dunet et al. [9] who found good quality images in $100 \%$ of the cases. This difference could be explained by the fact that our 
tracer of choice is the MIBI. Very few patients have benefited from Tetrofosmin. Indeed, studies have shown that the bioavailability of Tetrofosmin is more favorable than that of MIBI due to its rapid uptake by the myocardium, its rapid clearance, its liver fixation but also its weak digestive fixation [10] [11].

\subsection{Myocardial Scintigraphy and Pre-Test Probability}

TSMP was positive in all patients with a high pre-test probability. Patients with a low pre-test probability had a negative TSMP. And this was statistically significant. This confirms that myocardial scintigraphy should not be performed as a first-line treatment in patients with a low pre-test probability. According to the recommendations of the American Heart Association (A.H.A) and the American College of Cardiology (ACC), a diagnostic test for coronary artery disease is recognized as a class I indication only in patients with An intermediate pre-test probability. The indication for scintigraphy is only class IIb in patients with low or high pre-test probability. Considering the normality rate of TSMP corresponding to the normal examination rates among patients with a pre-test probability of less than $10 \%$, this specificity reaches $89 \%$. This high diagnostic performance makes TSMP at the opposite of ECG, an excellent diagnostic tool in patients with intermediate probability [12].

\subsection{Myocardial Scintigraphy and Transthoracic Echocardiography}

M.P.T.S was positive in $38.46 \%$ of patients with normal trans-thoracic echocardiography. This could be explained by the fact that the echocardiography carried out in the basal state allowed a sufficient perfusion of the myocardium to ensure its metabolic needs and thus the segmental and overall kinetics of the ventricle became normal. The effort made during myocardial scintigraphy results in an increase in myocardial oxygen consumption which is responsible in the presence of coronary lesions for M.P.T.S. Moreover, the anomalies of this perfusion are the first to appear during the ischemic cascade before any modification of the myocardial contractility.

\subsection{Abnormalities of Perfusion Myocardial Scintigraphy}

The M.P.T.S was positive in 28 patients $(66.66 \%)$. Of these patients 16 had pure ischemia, $57.14 \%$ (16/28), 7 patients had pure necrosis (25\%) and 5 patients (17.85\%) had both necrosis and ischemia. A positive scintigraphy accentuates the number of cardiovascular events per year which is multiplied by 7 . A metaanalysis of 39 studies with 69,655 patients for a mean follow-up of 2.3 years reported a rate of coronary events of $3 \%$ per year. In this series, those with a negative scintigraphy had a cardiovascular event rate of $0.8 \%$ /year and for those with moderate to severely positive infusion, the rate of coronary events was 5.9\%/ Year [13]. This risk of cardiovascular events is increased in proportion to the number of myocardial segments affected [14]. 


\section{Limitations of Our Study}

The M.P.T.S used alone has a definite interest in the diagnosis of coronary artery disease but does not predict prognosis. Its synchronization with E.C.G would allow a joint study of myocardial perfusion and an evaluation of left ventricular function. This modality is absent in our study and allows us to measure the volume of the left ventricle, its ejection fraction, its thickening and parietal kinetics [15]. This makes it possible to improve the specificity by highlighting the doubtful non-reversible defects. According to the above, this method allows a considerable time saving in the assessment and management of the coronary patient [16]. The left ventricular ejection fraction (L.V.E.F) is considered a major prognostic factor. According to Maurice Lévy et al. [17], when it is less than 45\% with a systolic V.T.E volume greater than $70 \mathrm{ml}$, it stratifies patients into high-risk groups. A VTS of less than $70 \mathrm{ml}$ is closely related to a low mortality (0.4\%/year), even in the case of severe hypoperfusion. A VTS greater than $70 \mathrm{ml}$ with severe or moderate hypoperfusion is associated with a higher mortality (2.4\% and 3.7\%/year). At the same L.V.E.F, a VTS greater than $70 \mathrm{ml}$ identified patients at higher risk than those with a VTS below $70 \mathrm{ml}$. Despite the smallness of our sample, the two-hour compact study of myocardial perfusion by TEMP with ${ }^{99 \mathrm{~m}} \mathrm{Tc}$-tetrofosmin synchronized with E.C.G with early post-stress acquisition could be introduced into our service. This would provide information on both myocardial perfusion and ventricular function while minimizing patient time in the department for good radiation protection [9]. The introduction of myocardial perfusion scintigraphy synchronized with the ECG and the use of thallium would improve the quality of our services by specifying the viability of the heart muscle.

\section{Conclusion}

Myocardial perfusion scintigraphy has an excellent cost-benefit ratio. The accessibility of coronary angiography in Senegal gives a renewed interest. Studies have shown that performing this non-invasive examination before invasive explorations revealed a number of indications of coronarography and revascularization divided by two as well as a reduced number of cardiac events in follow-up. When combined with other clinical and paraclinical elements, it provides valuable diagnostic and therapeutic guidance to the cardiologist in the management of coronary disease. Synchronized with E.C.G, M.P.T.S is a very sensitive means of diagnosing coronary artery disease, but also an important element in the assessment of prognosis and the selection of patients to benefit from coronary angiography.

\section{References}

[1] Angehrn, W. (2001) Chronic Ischemic Heart Disease. Forum Médical Suisse, 8, 1187-1191.

[2] Ferrario, M., Cesana, G. and Vanuzzo, D. (2001) Surveillance of Ischaemic Heart 
Disease: Results from the Italian Monico Population. International Journal of Epidemiology, 30, 23-29. https://doi.org/10.1093/ije/30.suppl_1.S23

[3] Murray, C.J. and Lopez, A.D. (1997) Alternative Projections of Mortality and Disability by Cause 1990-2020: Global Burden of Disease Study. The Lancet, 349, 1498-1504. https://doi.org/10.1016/S0140-6736(96)07492-2

[4] Ladenheim, M.L., Pollock, B.H., Rozanski, A., Berman, D.S., Stanilof, H.M., Forrester, J.S., et al. (1986) Extent and Severity of Myocardial Hypoperfusion as Predictors of Prognosis in Patients with Suspect Coronary Artery Disease. Journal of the American College of Cardiology, 7, 464-471. https://doi.org/10.1016/S0735-1097(86)80454-5

[5] Northridge, D.B., Grand, S., Henderson, E., Ray, S., Mcmurray, J. and Dargie, H.J. (1990) A Novel Exercise Protocol Suitable for Use on a Treadmill or a Bicycle Ergometer. British Heart Journal, 64, 313-316. https://doi.org/10.1136/hrt.64.5.313

[6] Diamond, G.A. and Forrester, J.S. (1979) Analysis of Probability in the Clinical Diagnosis of Coronary Artery Disease. The New England Journal of Medicine, 300, 1350-1358. https://doi.org/10.1056/NEJM197906143002402

[7] Sanmarco, M.E., Pontius, S. and Selvester, R.H. (1980) Abnormal Blood Pressure Response and Marked Ischemic St-Segment Depression as Predictors of Severe Coronary Artery Disease. Circulation, 61, 572-578.

https://doi.org/10.1161/01.CIR.61.3.572

[8] Depuey, E.G. (1994) How to Detect and Avoid Myocardial Perfusion SPECT Artifacts. The Journal of Nuclear Medicine, 35, 699-702.

[9] Dunet, V., Costo, S., Sabatier, R., Grollier, G., Bouvard, G. and Agostini, D. (2010) Feasibility and Diagnostic Accuracy of a Myocardial Scintigraphy Protocol Synchronized with E.C.G in Two Hours: The Myofaste Study. Nuclear Medicine, 34, 211-218.

[10] Jain, D., Wackers, F.J., Mattera, J., Mc Mahon, M., Sinusas, A.J. and Zaret, B.L. (1993) Biokinetics of Technetium-Tétrofosmine: Myocardial Perfusion Imaging Agent: Implications for a One-Day Imaging Protocol. The Journal of Nuclear Medicine, 34, 1254-1259.

[11] Flamen, P., Bossuyt, A. and Franken, P.R. (1995) Technetium-99m-Tetrofosmin in Dipyridamole-Myocardial Stress SPECT Imaging: Intraindividual Comparison with Technetium-99m-Sestamibi. The Journal of Nuclear Medicine, 36, 2009-2015.

[12] Vanzetto, G., Machecourt, J. and Fagret, D. (2000) Myocardial Perfusion Tomoscintigraphy (Mpts) and Prognostic Evaluation of Coronary Artery Disease. Review of the Acomen, 6, 114-121.

[13] Abdenbi, K. (2002) Smoking Cessation in the Coronary. Annales de Cardiologie et d'Angéiologie, 51, 357-366. https://doi.org/10.1016/S0003-3928(02)00148-8

[14] Worker, M.J., Bernard, M., Hitzel, A., Vera, P. and Manrique, A. (2009) Evaluation of Myocardial Sideration Post-Exertion Immediately by Tomoscintigraphy with 99mtc Coupled to the ECG. Nuclear Medicine, 33, 331-337.

[15] Manrique, A. and Marie, P.Y. (2003) Recommendations for the Performance and Interpretation of Myocardial Perfusion Tomoscintigraphy. Archives des Maladies du Coeur et des Vaisseaux, 96, 695-711.

[16] Calizzano, A. (2007) Current Position and Future Prospects of Myocardial Perfusion Scintigraphy. Nuclear Medicine, 31, 604-609.

[17] Levy, M. (2006) Myocardial Scintigraphy and Prognosis in Coronary Disease. MT Cardiologists, 2, 223-234. 